\title{
Predictors of smoking relapse in a cohort of adolescents and young adults in Monastir (Tunisia)
}

\author{
Sana El Mhamdi, Asma Sriha, Ines Bouanene, Arwa Ben Salah, Kamel Ben Salem and Mohamed Soussi Soltani
}

\begin{abstract}
Background: Smoking prevalence in adolescents and young adults is substantially elevated in Tunisia. Moreover, there is a lack of knowledge regarding the effectiveness and associated factors in smoking cessation interventions among adolescents and young adults. This study aims at identifying the major factors leading to smoking relapse among adolescents and young adults in the region of Monastir, Tunisia.

Methods: We carried out a prospective cohort study at the smoking cessation center of the University hospital of Monastir, Tunisia. The population study consisted of all adolescents and young adults (15-30 years) consulted during a period of two years (2009 - 2010). A questionnaire was used to explore the patient's sociodemographic characteristics, smoking history, nicotine dependence (Fagerstrom test) and anxiety / depression (Hospital Anxiety and Depression Scale). A telephone survey was conducted in July 2011 to assess smoking cessation results. A multivariate Cox regression was used to identify predictors of smoking relapses.

Results: A total of 221 adolescents and young adults were included in this study with a mean age of $25.5 \pm 3.9$ years. At follow up, 59 study participants (26.7\%) were abstinent and the overall median abstinence was 2 months. In the multivariate analysis smoking relapse was associated with being an adolescent patient (HR 2.16; 95\% Cl: 1.54-3.05), medium or higher nicotine dependence at baseline (HR 2.66, 95\% Cl: 1.06-7.05 and HR 3.12, 95\% Cl: 1.20-8.12 respectively), not receiving treatment ( $\mathrm{HR} 1.70,95 \% \mathrm{Cl}: 1.25-2.33)$ and have friend who is a smoker (HR 1.63; 95\% Cl: 0.96-2.79).

Conclusion: The results of this study provide important information about beneficial effect of smoking cessation support for adolescent and young adults. More efforts must be deployed to deal with contributing factors to smoking relapse.
\end{abstract}

\section{Introduction}

Smoking is one of the leading public health problems worldwide; it is one of the most preventable causes of death. In Tunisia, tobacco smoking use is substantially elevated. Adult smoking prevalence is estimated at $17.3 \%$ according to the "BREATHE study" [1]. This rate remains higher among adolescents rather than adults (from 16 to 29.2\%) [2,3]. In addition, cultural factors such as parents who smoke, doctors, nurses, and teachers who smoke in the workplace may add to the creation of a pro-smoking environment [4].

Research suggests that knowledge about the health effects of smoking is a necessary component of tobacco control measures and can result in a postponement of

\footnotetext{
* Correspondence: sanaelmhamdi@gmail.com

Smoking Cessation Center of Monastir University Hospital, Monastir, Tunisia
}

initiation and smoking cessation in youth [5]. Smoking cessation counseling is widely recommended by national and international health organizations, which underscore the great potential for a beneficial intervention at the primary care level. However, smoking cessation outcomes may be affected by smoker characteristics and life context variables [6]. Thus the objective of our study was to identify predictors of smoking relapse among adolescents and young adults in the region of Monastir, Tunisia.

\section{Methods}

Design

We conducted a prospective cohort study in the city of Monastir in Tunisia from 01/01/2009 to 31/12/2010. The study was carried out in the center of smoking cessation of Monastir University Hospital. All patients aged 15 to 30 years were included in the study, independently of the
() Biomed Central

(c) 2013 El Mhamdi et al.; licensee BioMed Central Ltd. This is an Open Access article distributed under the terms of the Creative Commons Attribution License (http://creativecommons.org/licenses/by/2.0), which permits unrestricted use, distribution, and reproduction in any medium, provided the original work is properly cited. 
number of cigarettes smoked per day, number of packyears or number of previous attempts to quit. All patients were informed and accepted that their data will be used for scientific research. We obtained the approval of the ethical committee of the University Hospital of Monastir (Tunisia).

\section{Intervention}

All smokers received a standardized program for smoking cessation including behavioral counseling and pharmacological treatment. The behavioral counseling consisted of a 45-minute session dealing with aspects of smokingrelated morbidity and mortality, key issues of nicotine addiction and the development of a specific action plan for stoppingsmoking. Pharmacotherapy consists of Nicotine Replacement Therapy (NRT), Varenicline (VAR) and bupropion (BUP) and was assigned depending on availability (supplied by the public health system, free of charge) and patient comorbidities and smoking dependence. Pharmacological therapy was prescribed for at least 12 weeks. Patients attended follow-up control visits at weeks 2, 4, 8 and 12 after the scheduled quit day and monthly until the sixth month. Abstinence exceeding sixth months was assessed by telephone survey.

The following two definitions were used in the study:

Quit date: starting date of smoking abstinence. This date is chosen by the patient himself

Point date: date of the last assessment of smoking status. In this study the point date was set on September 15, 2011.

At baseline a detailed smoking history and health data, was gathered. The questionnaire included items on: Sociodemographic characters (gender, current age, current activity, age of cigarette initiation, age of regular smoking and motivation behind their smoking habit); Daily number of cigarettes and level of smoking addiction using the French version of the Fagerström test for nicotine dependence (FTND) [7] divided into very low dependence $(0-2)$, Low / Medium dependence (3 - 6) and high dependence (7 - 10); Anxiety and depressive behavior using the French version of the Hospital Anxiety and Depression Scale (HAD) [8]. A HAD score $\geq 11$ corresponds to anxiety or depressive disorders; Friends that are smokers (the investigators decide to consider at least one friend who is smokers in the patient friend's group; Moderate alcohol consumption (one drink or less per day for women and two drinks or less per day for men during the survey [9]); Number of visits to the medical center and use of pharmacological treatment (corresponding to the use of NRT, VAR and BUP).

At follow up the patient's smoking status was determined by phone calls focus on the following topics: the current status of smoking, the date of relapse and the causes associated with it. Moreover, self-reported cigarette consumption and possible adverse effects were notified at all follow-up visits.

\section{Statistical analysis}

In the descriptive analysis, categorical variables are expressed as proportions and continuous variables as mean (with standard deviation). Univariate (crude) analysis of variables from the entire population was performed using $\chi^{2}$ test and two-sample test for continuous data. Data were expressed as an odds ratio and $95 \%$ confidence interval. All tests used a significant level of 0.05 . In this longitudinal study participants could make quit attempts at different times during the follow-up, and thus they also may have different lengths of follow-up to observe relapse. In this case we used a multivariate survival analyses (Cox regression) to identify predictors of relapses. This method allows for differential follow-up and efficiently accommodates missing data due to censoring. Models were fitted, including all factors associated with the outcome, in a stepwise procedure by Kaplan-Meier method. Factors were included in the model when $\mathrm{p} \leq 0.25$. To be introduced in the multivariate analysis the cutoff point of variables using a visual analogue scale (motivations of smoking behavior) were defined according to ROC curve.

Data was analyzed by SPSS 17.0.

\section{Results}

\section{Characteristics of the study sample}

Among the attendees of the smoking cessation center, 221 adolescents and young adults were tracked during a period of 24 months (from January 2009 to December 2010).

The study sample included 210 men (95\%) and 11 women $(5 \%)$. The patient's mean age was 25.5 years (SD 3.9). The mean age of smoking initiation was 15.2 years (SD 3.4). A third of our patients $(n=80)$ consumed alcoholic drinks occasionally (once a month). The mean number of daily cigarette use among our patients was 26 (SD 10.4 ) with a mean FTND about 6.4 (SD 2). Mean scores for anxiety and depression were 8.4 (SD 4.2) and 5 (SD 3.2) respectively. Basic information and demographic characteristics of the participants were reported in Table 1.

As depicted in Table 2, several motivations for smoking behavior were assessed using a visual analog scale from 0 to 10. The main motivation for the use of cigarettes was to "deal with stress" with a mean of 7.4(SD 3). Other motivations include automatism with a mean of 7.1 (SD 3.2) and moral support with a mean of 7.3 (SD 3.3).

\section{Predictors of smoking relapse}

At follow up, 59 patients (26.7\%) were abstinent at the study endpoint with a median abstinence delay of 2 months (95\% CI [1.63 - 2.37]). The median delay to smoking relapse was 0.75 months $(95 \% \mathrm{CI}[0.42-1.07])$ for adolescents and 2.50 months $(95 \% \mathrm{CI}[2.14-2.86])$ for young adults $(\mathrm{p}<0.01)$. Table 3 presents the association of each studied factor with the delay of a quit attempt. 
Table 1 Sociodemographic characteristics of the study sample at baseline $(\mathrm{N}=\mathbf{2 2 1})$

\begin{tabular}{|c|c|c|}
\hline Factors & Number (\%) & Means \pm SD \\
\hline Age (years) & - & $25.5 \pm 3.9$ \\
\hline Gender & & - \\
\hline Female & $11(5)$ & \\
\hline Male & $210(95)$ & \\
\hline Current activity & & - \\
\hline Profession/training & $201(90.9)$ & \\
\hline Unemployed & $20(9.1)$ & \\
\hline Age of smoking initiation & - & $15.2 \pm 3.4$ \\
\hline Age of regular smoking & - & $18.5 \pm 3.4$ \\
\hline Current alcohol use & & - \\
\hline Yes & $80(36.2)$ & \\
\hline No & $141(63.8)$ & \\
\hline Fagerström score & - & $6.4 \pm 2$ \\
\hline $\begin{array}{l}\text { Daily number of cigarettes } \\
\text { smoked }\end{array}$ & - & $26 \pm 10.4$ \\
\hline Anxiety score & - & $8.4 \pm 4.2$ \\
\hline Depression score & - & $5 \pm 3.2$ \\
\hline Have friends that are smokers & & - \\
\hline Yes & $64(29)$ & \\
\hline No & $157(71)$ & \\
\hline $\begin{array}{l}\text { Number of visits to the medical } \\
\text { center for treatment }\end{array}$ & & - \\
\hline$<3$ & $70(31.7)$ & \\
\hline 3 visits or more & $151(68.3)$ & \\
\hline Use of pharmacological treatment & & - \\
\hline No & $7(3.2)$ & \\
\hline Yes & $214(96.8)$ & \\
\hline
\end{tabular}

In the multivariate analysis in Table 4, smoking relapse was associated with being an adolescent patient (HR 2.16; 95\% C.I: 1.54-3.05), medium or higher nicotine dependence at baseline (HR 2.66, 95\% CI: 1.06-7.05 and HR 3.12, 95\% CI: 1.20-8.12 respectively), less than three visits to the medical center, having not received

Table 2 Distribution of participants according to the motivation behind their smoking behavior

\begin{tabular}{lccc}
\hline Motivation (n) & Mean (SD) & Minimum & Maximum \\
\hline Automatic gesture (199) & $7.1(3.2)$ & 2 & 10 \\
Conviviality (192) & $4.8(3.8)$ & 0 & 10 \\
Enjoyment (189) & $5.4(3.3)$ & 0 & 10 \\
Dealing with stress (188) & $7.4(3.0)$ & 1 & 10 \\
Concentration (201) & $6.3(3.4)$ & 0 & 10 \\
Moral support (186) & $7.3(3.3)$ & 0 & 10 \\
Fighting obesity (179) & $2.2(0.7)$ & 0 & 7 \\
\hline
\end{tabular}

treatment (HR 1.70, 95\% CI: 1.25-2.33) and have friend who is a smoker (HR 1.63; 95\% CI: 0.96-2.79).

\section{Discussion}

In this study we used data from the Smoking Cessation Center of Monastir University Hospital. Among the 310 monitored smokers, 221 answered the phone survey and the prevalence of abstinence at the endpoint was $26.7 \%$. This kind of center validated secondary prevention strategies to help people quit smoking [10]. This rate is similar to those reported in literature for adolescents and young adults $[11,12]$. According to literature, younger adults were more likely to be successful quitters than adolescents [13]. In our study we also identified that adolescence may be related to smoking relapses.

A number of studies have indicated that the likelihood of smoking cessation is greater in smokers who had initiated cigarette smoking after the age of 13 , in comparison to those who had begun earlier [14]. In our study, the age of smoking initiation as well as the age of regular smoking was not identified as predictor of smoking relapses. The effects of these two ages were perhaps more important in adulthood rather than adolescence among Tunisian youth.

Other findings from this study suggest that smoking cessation might vary also by the level of nicotine dependence. Highly and moderately dependent smokers were found to be less likely to quit than lower dependent ones. Indeed research has indicated that higher nicotine dependence in adolescents and young adulthood predicts later dependence and smoking relapses [15]. Thus, quitting smoking as an adolescent or young adult may substantially alter the risk for later tobacco dependence.

Research has indicated that decreases in the proportion of friends who smoke were robust predictors of cessation [16]. The likelihood of quitting among young people is potentially dependent on the extent of smoking among their peers. Behavioral interventions based on peer education with social networks can help smoking cessation efforts [17].

In our study, having a friend who smoked was not a strongly predictor of smoking relapse. These findings have also been reported by other African countries. For example, Egyptian adolescents were noted to be more influenced by their family's smoking behavior and perceived adult smoking norms than their peers' smoking behavior [18].

It is recognized that smoking cessation can be achieved with or without assistance from healthcare but pharmacotherapy may be better in smoking cessation than self-help [19]. NRT and VAR therapy are noted to be effective strategies in smoking cessation especially among adults. Studies proved that they increase the individual's chance of successfully quitting smoking [20]. Our study, as recent 
Table 3 Unadjusted delay in smoking relapse among respondents at follow up $(\mathrm{N}=\mathbf{2 2 1})$

\begin{tabular}{lccc}
\hline Factors & $\begin{array}{c}\text { Median delay } \\
\text { (months) }\end{array}$ & $95 \% \mathrm{Cl}$ & $\mathrm{p}$-value \\
\hline
\end{tabular}

Age
Adolescent
Young adult
Gender
Female
Male
Current activity
Permanent profession/
training
Unemployed

Age at smoking initiation

$<13$ years of age

$\geq 13$ years of age

Age at regular smoking

$$
<18 \text { years of age }
$$$$
\geq 18 \text { years of age }
$$

Current alcohol use

Yes

FTND

Very low dependence

Low / Medium dependence

High dependence

$\begin{array}{cc}0.75 & 0.42-1.07 \\ 2.5 & 2.14-2.86 \\ & \\ 2.0 & 1.60-2.40 \\ 1.8 & 0.70-3.30\end{array}$

$<0.01$

(1)

0.52

2.0

$2.0 \quad 1.60-2.39$

0.70

$1.9 \quad 0.70-3.29$

$.2 \quad 0.87-1.84$

$2.0 \quad 1.61-2.38$

0.21

$.5 \quad 0.88-2.11$

$2.5 \quad 2.10-2.89$

0.31

2.0

$1.07-2.92$

3.0

$1.80-4.19$

0.22

aily cigarette use

$<20$

$\geq 20$

$18.5 \quad 9.30-$

25.20

$3.0 \quad 0.85-5.14$

$2.0 \quad 1.40-2.60$

2.0

$1.71-3.82$

$1.58-2.41$

Anxiety

Yes

No

2.0

2.5

$1.01-2.98$

$2.01-2.98$

\section{Depression}

Yes

No

Smoking friends

$\begin{array}{lll}\text { Yes } & 1.5 & 0.80-2.19 \\ \text { No } & 3.0 & 2.60-3.40\end{array}$

Number of visits to the medical center

$\begin{array}{lll}<3 & 1.0 & 0.63-1.36 \\ 3 \text { or more } & 3.0 & 1.25-5.66\end{array}$

Use of pharmacological treatment

$\begin{array}{lll}\text { No } & 4.0 & 2.62-9.13 \\ \text { Yes } & 2.0 & 1.63-2.37\end{array}$

0.011
Table 4 Factors associated with relapses delay in smoking cessation

\begin{tabular}{|c|c|c|c|}
\hline Factors & $\mathrm{HR}$ & $95 \% \mathrm{Cl}$ & $p$ \\
\hline \multicolumn{3}{|l|}{ Fagerström nicotine dependence } & 0.040 \\
\hline Very low dependence & 1 & - & \\
\hline Low / Medium dependence & 2.66 & $1.06-7.05$ & \\
\hline High dependence & 3.12 & $1.20-8.12$ & \\
\hline \multicolumn{3}{|l|}{ Patient's age } & 0.038 \\
\hline Young adult & 1 & - & \\
\hline Adolescent & 2.16 & $1.54-3.05$ & \\
\hline \multicolumn{3}{|c|}{ Number of visits to the medical center } & $<0.001$ \\
\hline$<3$ & 1 & - & \\
\hline 3 visits or more & 0.46 & $0.33-0.64$ & \\
\hline \multicolumn{3}{|l|}{ Smoking friends } & 0.060 \\
\hline No & 1 & - & \\
\hline Yes & 1.63 & $0.96-2.79$ & \\
\hline \multicolumn{3}{|l|}{ Using treatment } & $<0.001$ \\
\hline Yes & 1 & - & \\
\hline No & 1.70 & $1.25-2.33$ & \\
\hline
\end{tabular}

Results of a multivariate Cox regression controlling for gender.

studies [21], proved the effectiveness of smoking cessation therapies in younger smokers. Other studies in developed countries showed that the mean number of cigarettes smoked daily among adolescents and young adults may not exceed 8 or 9 cigarettes per day [22]. Our data showed a higher dependent adolescent population with a mean number of 26 cigarettes smoked daily. With regard to this result a better availability of professional cessation services directed to younger smokers and availability of pharmacological treatment potentially is needed in Tunisia as both treatment and regular follow-up increase long term smoking quitting [23]. A successful treatment may require close follow up, behavioral support and pharmacological therapy [24]. Compared to other developing countries (especially North African countries) current smoking prevalence of Tunisian adolescents is higher (15.3\%) and initiation susceptibility reaches $25 \%$ among boys thus understanding contributors to smoking initiation and cessation is important for public health interventions [25].

\section{Limitations and strengths}

The important strength of this study is its prospective character. We performed a prospective cohort study over a period of two years to identify predictors of smoking relapse among adolescents and young adults. The advantage of the prospective design is the relative insensitivity for selection and information bias compared with a casecontrol design. Second, the survival "Cox Regression" allows us to take full account of the censored data. There are also some limitations to our study. The first is the 
lack of nicotine biomarkers in our context (especially hair and nails analysis) and self-reported smoking cessation. Second, the small number of women number does not allow investigation of interaction and separate analyses for men and women.

\section{Conclusion}

The results of this study confirm the beneficial effect of smoking cessation support for adolescent and young adults. More efforts must be deployed to deal with contributing factors to smoking relapse. Furthermore, health education programmes regarding this potential harm of tobacco use should be implemented for young people.

\section{Consent}

Written informed consent was obtained from the patient for publication of this report and any accompanying files or images.

\section{Competing interests}

The authors declare that they have no competing interests.

\section{Authors' contributions}

SEM: participate in the redaction of the reaserch protocol / participate in the patient's follow-up / perform the statistical analysis / write the manuscript. AS: responsable of the patient's follow-up. IB: participate in the redaction of the reaserch protocol. ABS: participate in the statistical analysis / participate in patient's follow-up. KBS: responsable of the corrections of the reaserch protocol and statistical analysis. MSS: responsable of the coordination of the reaserch group. All authors read and approved the final manuscript.

\section{Acknowledgments}

We thank all members of the department of Preventive Medicine and Epidemiology for their helpfulness.

Received: 16 January 2013 Accepted: 15 May 2013

Published: 25 May 2013

\section{References}

1. Idrees M, Koniski ML, Taright S, et al: Smoking habits in the Middle East and North Africa: results of the BREATHE study. Respir Med 2012, 106(Suppl 2):S33-44.

2. Fakhfakh R, Hsairi M, Achour N: Epidemiology and prevention of tobacco use in Tunisia: a review. Prev Med 2005, 40:652-7.

3. El Mhamdi S, Wolfcarius-Khiari G, Mhalla S, Ben Salem K, Soltani MS: Prevalence and predictors of smoking among adolescent schoolchildren in Monastir, Tunisia. East Mediterr Health J 2011, 17:523-8.

4. Osinubi OY, Slade J: Tobacco in the workplace. Occup Med 2002, 17:137-58

5. Brown J: A review of the evidence on technology-based interventions for the treatment of tobacco dependence in college health. Worldviews Evid Based Nurs 2013, 19. In press.

6. Zhou X, Nonnemaker J, Sherrill B, Gilsenan AW, Coste F, West R: Attempts to quit smoking and relapse: factors associated with success or failure from the ATTEMPT cohort study. Addict Behav 2009, 34:365-73.

7. Etter JF: A comparison of the content-, construct- and predictive validity of the cigarette dependence scale and the Fagerström test for nicotine dependence. Drug Alcohol Depend 2005, 77:259-68.

8. Bjelland I, Dahl AA, Haug TT, Neckelmann D: The validity of the Hospital Anxiety and Depression Scale. An updated literature review. J Psychosom Res 2002, 52:69-77.

9. Moore D, Aveyard P, Connock M, Wang D, Fry-Smith A, Barton P. Effectiveness and safety of nicotine replacement therapy assisted reduction to stop smoking: systematic review and meta-analysis. BMJ 2009, 338:B1024.

10. El-Sharkawy GF: Cigarette smoking among University students: family related and personal risk factors. J Am Science 2011, 7:260-8.
11. Ayo-Yusuf OA, Szymanski B: Factors associated with smoking cessation in South Africa. S Afr Med J 2010, 100:175-179.

12. Audrain-McGovern J, Halbert CH, Rodriguez D, Epstein LH, Tercyak KP: Predictors of participation in a smoking cessation program among young adult smokers. Cancer Epidemiol Biomarkers Prev 2007, 16:617-619.

13. Breslau N, Peterson EL: Smoking cessation in young adults: age at initiation of cigarette smoking and other suspected influences. Am J Public Health 1996, 86:214-20.

14. Van De Ven MO, Greenwood PA, Engels RC, Olsson CA, Patton GC: Patterns of adolescent smoking and later nicotine dependence in young adults: a 10-year prospective study. Public Health 2010, 124:65-70.

15. Cengelli S, O'Loughlin J, Lauzon B, Cornuz J: A systematic review of longitudinal population-based studies on the predictors of smoking cessation in adolescent and young adult smokers. Tob Control 2012. 21:355-62.

16. Klatt C, Berg CJ, Thomas $\mathrm{J}$, et al: The role of peer E-mail support as part of a College smoking-cessation website. Am J Prev Med 2008, 35(6 Suppl):S471-8.

17. Islam SM, Johnson CA: Influence of known psychosocial smoking risk factors on Egyptian adolescents' cigarette smoking behavior. Health Promot Int 2005, 20:135-45.

18. Grimshaw GM, Stanton A: Tobacco cessation interventions for young people. Cochrane Database Syst Rev 2006, 18, CD003289.

19. Kralikova E, Kozak JT, Rasmussen T, Gustavsson G, Le Houezec J: Smoking cessation or reduction with nicotine replacement therapy: a placebo-controlled double blind trial with nicotine gum and inhaler. BMC Publ Health 2009, 9:433.

20. Toljamo T, Hamari A, Nieminen P, Kinnula VL: Young male daily smokers are nicotine dependent and experience several unsuccessful quit attempts. Scand J Prim Health Care 2012, 30:183-8.

21. Rath JM, Villanti AC, Abrams DB, Vallone DM: Patterns of tobacco use and dual use in US young adults: the missing link between youth prevention and adult cessation. J Environ Public Health 2012, 2012:679134.

22. West R, McNeill A, Raw M: Smoking cessation guidelines for health professionals: anupdate. Health Education Authority. Thorax 2000, 55:987-99.

23. Avila-Tang E, Apelberg BJ, Yamaguchi N, Katanoda K, Sobue T, Samet JM: Modelling the health benefits of smoking cessation in Japan. Tob Control 2009, 18:10-7

24. Madkour AS, Ledford EC, Andersen L, Johnson CC: Tobacco advertising/ promotions and adolescents' smoking risk in Northern Africa. Tob Control 2013, 8. Article in press.

25. Florescu A, Ferrence R, Einarson T, Selby P, Soldin O, Koren G: Methods for quantification of exposure to cigarette smoking and environmental tobacco smoke: focus on developmental toxicology. Ther Drug Monit 2009, 31:14-30.

doi:10.1186/1617-9625-11-12

Cite this article as: El Mhamdi et al:: Predictors of smoking relapse in a cohort of adolescents and young adults in Monastir (Tunisia). Tobacco Induced Diseases 2013 11:12.

\section{Submit your next manuscript to BioMed Central and take full advantage of:}

- Convenient online submission

- Thorough peer review

- No space constraints or color figure charges

- Immediate publication on acceptance

- Inclusion in PubMed, CAS, Scopus and Google Scholar

- Research which is freely available for redistribution 